\title{
UMA APLICAÇÃO DO MÉTODO FITRADEOFF NA COMPARAÇÃO DE PODERES COMBATENTES DE UNIDADES DE SUPERFÍCIE
}

\author{
Raphael Corrêa Silva \\ Escola de Guerra Naval \\ Setor de Operações Navais \\ raphacorreasilva@gmail.com
}
Leonardo Antonio Monteiro Pessôa
Centro de Análises de Sistemas Navais
Departamento de Engenharia de Sistemas
lampessoa@terra.com.br

\section{Rodrigo José Pires Ferreira}

Universidade Federal de Pernambuco

Centro de Desenvolvimento em Sistemas de Informação e Decisão rodrigo@cdsid.org.br

\section{Helder Gomes Costa}

Universidade Federal Fluminense

Programa de Engenharia de Produção

heldergc@id.uff.br

\section{Adiel Teixeira de Almeida}

Universidade Federal de Pernambuco

Centro de Desenvolvimento em Sistemas de Informação e Decisão

almeida@cdsid.org.br

\section{RESUMO}

Este artigo apresenta uma aplicação da metodologia multicritério FITradeoff para o auxílio na Comparação de Poderes Combatentes (CPC) entre unidades navais de superfície. A metodologia utilizada permite, com auxílio de especialistas, efetuar uma comparação coerente entre diferentes unidades, possibilitando mesmo em face de reduzido tempo e recursos, contribuir de maneira efetiva para o planejamento militar.

Palavra-chave: Planejamento Militar; Multicritério; FITradeoff

\section{ABSTRACT}

This paper presents the use of FITradeoff methodology on the Comparison of the Combatant Power of naval units for Surface warfare. The method aids to perform a fast 
and coherent comparison, using the expert knowledge of a navy officer, contributing in an effective manner to a military operation planning, using reduced resources.

Keywords: Military Planning; Multiple Criteria; FITradeoff

\section{Como Citar:}

CORRÊA SILVA, Raphael; PESSÔA, Leonardo Antonio Monteiro; FERREIRA, Rodrigo José Pires; COSTA, Helder Gomes; ALMEIDA, Adiel Teixeira. Uma aplicação do método FITradeoff na Comparação de Poderes Combatentes de unidades de superfície. In: SIMPÓSIO DE PESQUISA OPERACIONAL E LOGÍSTICA DA MARINHA, 19., 2019, Rio de Janeiro, RJ. Anais [...]. Rio de Janeiro: Centro de Análises de Sistemas Navais, 2019.

\section{INTRODUÇÃO}

O presente trabalho objetiva demonstrar a possibilidade de utilização de metodologia multicritério para auxiliar na Comparação de Poderes Combatentes (CPC), um dos passos importantes do planejamento militar, utilizando como prova de conceito a comparação entre unidades navais de superfície. A situação considerada para a definição dos parâmetros para o emprego da ferramenta foi a seguinte:

- Navios escoltas;

- Grupamentos Operativos conduzindo Ações de Superfície entre si; e

- Local de engajamento entre os Grupamentos Operativos com águas profundas e distante de qualquer litoral, i.e. arquipelágico ou continental.

Pretende-se neste trabalho a apresentação de uma proposta de utilização de metologia multicritério, de forma a auxiliar um tomador de decisão na comparação de poderes combatentes, pela geração de uma função de preferência. Desta forma, permitindo a avaliação, e comparação, de unidades de superfície, com base em suas características técnicas.

Para a execução a contento é necessária a participação de especialista, que fará o papel de decisor. Esta comparação será embasada na em uma escala pessoal de valoração definida pelos especialistas que compõem o Estado-Maior, sem excluir os aspectos subjetivos que envolvem a análise da situação, mas incorporando-os à estrutura de formalização.

De modo a caracterizar metodologicamente a estrutura do trabalho, a próxima seção apresenta um breve embasamento teórico. Este contempla alguns aspectos de métodos multicritério de auxílio à decisão, e detalha a abordagem empregada na condução do presente trabalho. A seção 3 apresenta os resultados, com foco nas referências identificadas. Por fim, a seção 4 traz uma breve discussão dos resultados e a conclusão deste trabalho. 


\section{METODOLOGIA}

A utilização de metodologias multicritério em problemas da esfera militar não é recente. Segundo [1], os exemplos são diversos, englobando principalmente aspectos logísticos e administrativos da esfera militar, com grande concentração de uso em metodologia Analytic Hierarchy Process (AHP), produzidos por pesquisadores ligados a institutos da China e dos Estados Unidos.

Entretanto desenvolvimentos mais diretamente focados ao planejamento militar, sobretudo nos campos tático e operacional são substancialmentes limitados, notando-se em sua constituição exploração ou de maior diversidade de métodos ou de articulações metodológicas.

\subsection{FITradeoff}

O presente trabalho vê a utilização do método FITradeoff, que é de recente criação [2], já explorado em problemas relacionados à esfera militar, por exemplo em [3] e [4], relacionados ao problema de escolha de armamento, como uma oportunidade de utilização em problema afeto diretamente ao planejamento militar, apresentando um ineditismo temático.

Como vantagens explicitadas para a utilização do método tem-se que [2]:

- o processo de utilização do método conta com um programa de auxílio a decisão

- sua configuração reduz o esforço cognitivo para o decisor

- seu processo tende a reduzir as inconsistências

Para este teste de conceito foi utilizado o modelo "FITradeoff escolha", que apresenta em ordem geral dois grupos de procedimentos. O primeiro diz respeito à ordenação por prioridade dos critérios, possibilitando a formação de um primeiro conjunto de inequações entre as constantes de escala.

O segundo passo consiste na elicitação flexível que gera alternativas diferentes entre si em apenas dois critérios. Apresenta-se para o decisor de maneira visual os desempenhos das alterativas geradas. O decisor pode então assinalar a sua preferência, segundo a sua experiência ou fruto das análises do Processo do Planejamento Militar/Processo do Planejamento Conjunto, na seguinte forma:

- pela alternativa A;

- pela alternativa B;

- considerar ambas indiferentes; ou

- declarar inconsistência. 
O processo continua até haver uma alternativa definida como superior ou até que o decisor considere o conjunto de opções restantes suficientemente limitado para estabelecer sua decisão.

No caso analisado neste trabalho,com base no julgamento de especialista, (emulando o papel de Comandante de parcela Naval de uma Teatro de Operações), o que se pretende é utilizar a metodologia FITradeoff para poder efetuar a valoração de trade-off entre critérios relevantes de avaliação, e assim construir uma função de preferência que permita comparação entre diferentes meios de superfície.

Desta forma este uso possibilita uma comparação quantitativa, mas com a incorporação de subjetividade do decisor no julgamento de valor, incorporando a experiência de decisor.

A escolha pela modalidade de escolha do FITradeoff tem a intenção de reduzir o número de questões a serem apresentadas ao decisor, apresentando uma limitação aos pesos para a definição de constantes de escala adequadas para a constituição da função de preferência.

Esta modalidade de escolha pode recair sobre as melhores constantes de escala para a alternativa definida como a escolha, ou havendo sugestões de constantes de escala iguais a zero, o que não é desejável para a finalidade da CPC, a arbitragem de constantes de escala entre os limites apresentados, mantendo um comportamento proporcional.

Cabe ressaltar, que as alternativas utilizadas como base para o estudo de caso são baseadas em dados quantitativos de meios navais de superfície, não existentes, mas simulados com proximidade suficiente à realidade.

\subsection{CPC e a Metododologia de Planejamento Militar}

A comparação de Poderes Combatentes (CPC) é um estudo que tem por objetivo contribuir para obter, primordialmente, uma avaliação quantitativa dos meios necessários para oposição à força inimiga estabelecendo conclusões sobre a própria força. [5]

Neste trabalho, é abordado o item de forças combatentes, podendo analisarse os outros itens componentes (Logística) [6] em futuros trabalhos. Especificamente devota-se neste trabalho a comparação sobre unidades de superfície de forças navais, utilizando-se para tal os seguintes eixos principais: mobilidade; detecção; e engajamento.

Estes três eixos desdobram-se em critérios, os quais somados à capacidade de guerra eletrônica (GE) e à possibilidade de aviação embarcada, permitirão a comparação entre aspectos críticos. O processo de desdobramento destas capacidades em aspectos operacionais críticos é coadunante com a metodologia de Avaliação Operacional.

A figura 1 apresenta um mapa conceitual levando em consideração diversos 
empregos de unidades de superfície. Note-se que a capacidade de GE e de Aviação orgânica, são relacionadas aos eixos de Detecção e Engajamento. Todavia, no modelo são tratadas separadamente, de modo que não haja superposição de critérios.

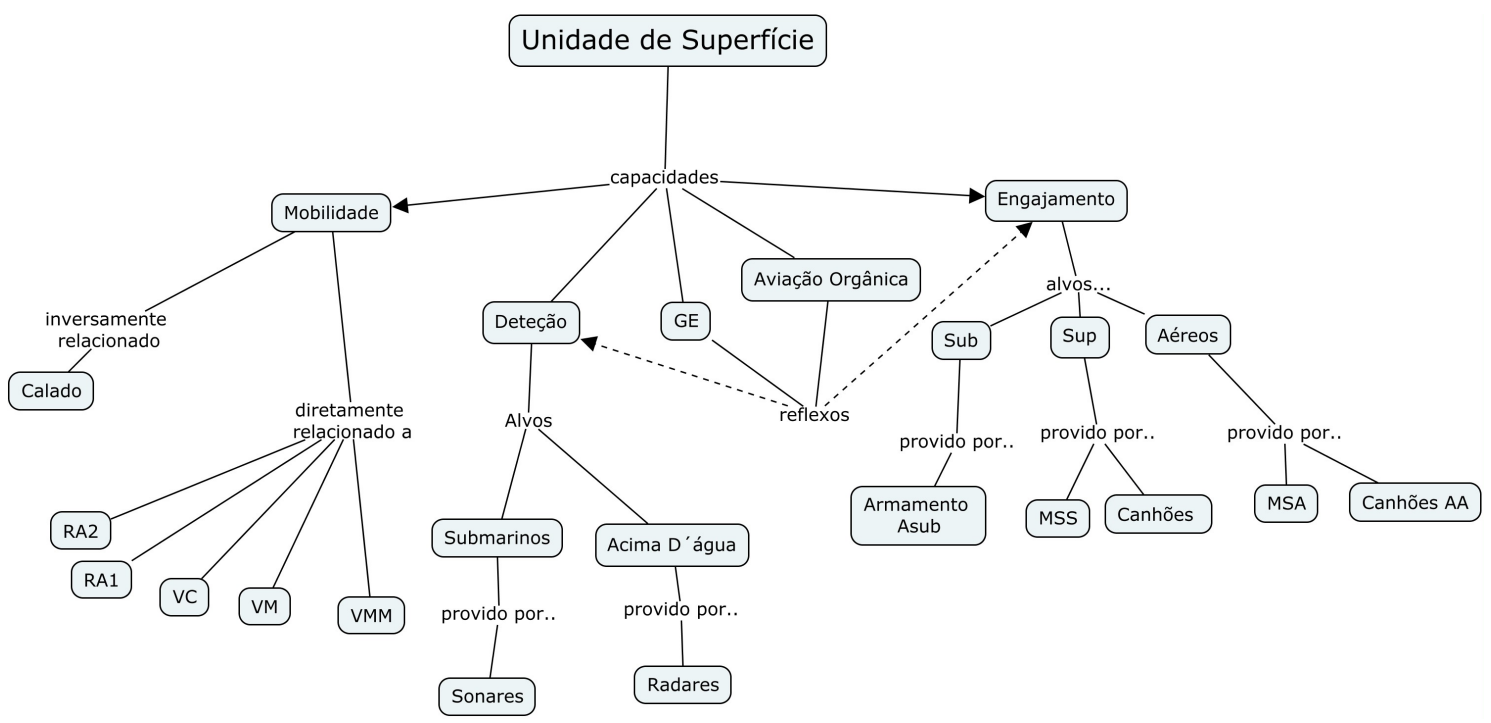

Figura 1: Estrutura conceitual dos critérios

De maneira geral seriam considerados os seguintes critérios para cada eixo:

- Mobilidade

Calado;

VC (Velocidade de Cruzeiro)

VMM (Velocidade Máxima Mantida)

VM (Velocidade Máxima)

RA1 (Raio de Ação em VC)

RA2 (Raio de Ação em VMM)

- Deteção

Sonares

Radares

- Engajamentos

Submarino (torpedos e armas submarinas)

Superfície (mísseis e canhões)

Aéreos (mísseis e canhões)

- GE

- Aeronave orgânica (quantidade) 
Este trabalho apresenta uma prova de conceito, e assim uma simplificação quanto à análise dos equipamentos de Deteção, GE e Engajamento. Seria ideal que cada equipamento fosse analisado em face de suas características de desempenho operacional, o que se consolida como possibilidade de extensão futura.

Por outro lado, a CPC também tem sua realização condicionada a um objetivo específico. Isto é, à luz da missão determinada, as mesmas unidades de superfície podem ser comparadas de maneira coadunante ao seu emprego em relação a meios de superfície, submarinos ou aéreos.

Outrossim, o modelo apresenta similaridade a uma estrutura hierárquica de critérios, presente em metodologias multicritério como o AHP. A conjectura de uma utilização hierárquica no FITradeoff já é defendida em [7].

A avaliação iniciada pelos níveis menos elevados da hierarquia é proposital, visando proporcionar ao tomador de decisão, a familiarização com os escores finais dos critérios, e permitir relacioná-los aos valores obtidos pelas alternativas, de modo a possibilitar a comparação intercritérios.

\section{RESULTADOS}

Os dados de conjunto de teste são listados a partir de meios simulados, com características verossímeis de emprego, e por questão de aspectos de confidencialidade são mencionados como 15 alternativas. O objetivo escolhido neste teste conceitual envolve a CPC de meios de superfície voltado para ações de superfícies. Desta forma, o diagrama inicial de eixos tem uma simplificação quanto aos critérios.

Para este trabalho, a análise está restrita à avaliação de emprego de unidades de superfície, obedecendo os conceitos atinentes as "Ações de Superfície" com o emprego exclusivo de navios escoltas. Assim sendo parcela da estrutura de critérios (relativos aos ambientes submarino e aéreo) não são incluídas neste trabalho. Da mesma forma, são excluídas as avaliações quanto aos sonares, as capacidades de engajamento aérea e submarina. Estes podem ser tratados em outros trabalhos.

Quanto ao eixo mobilidade, o critério de calado é analisado somente em termos de adequabilidade, estando sujeitos ao veto de alternativas inadequadas, portanto este foi excluído da análise.

A figura 2 apresenta a estrutura simplificada dos critérios para este estudo de caso.

Adicionalmente, as capacidades radar, GE e engajamento são tratadas neste trabalho de forma que os equipamentos disponíveis por cada alternativa sejam considerados critérios independentes. Não obstante ser desejável a análise mais profunda acerca dos equipamentos por desempenho, considera-se que seria uma simplificação desejável nesta etapa da pesquisa.

Também, alguns destes critério são tratados como variáveis contínuas, mesmo para os casos de presença/ausência do equipamento. Nestes casos a interpretação 


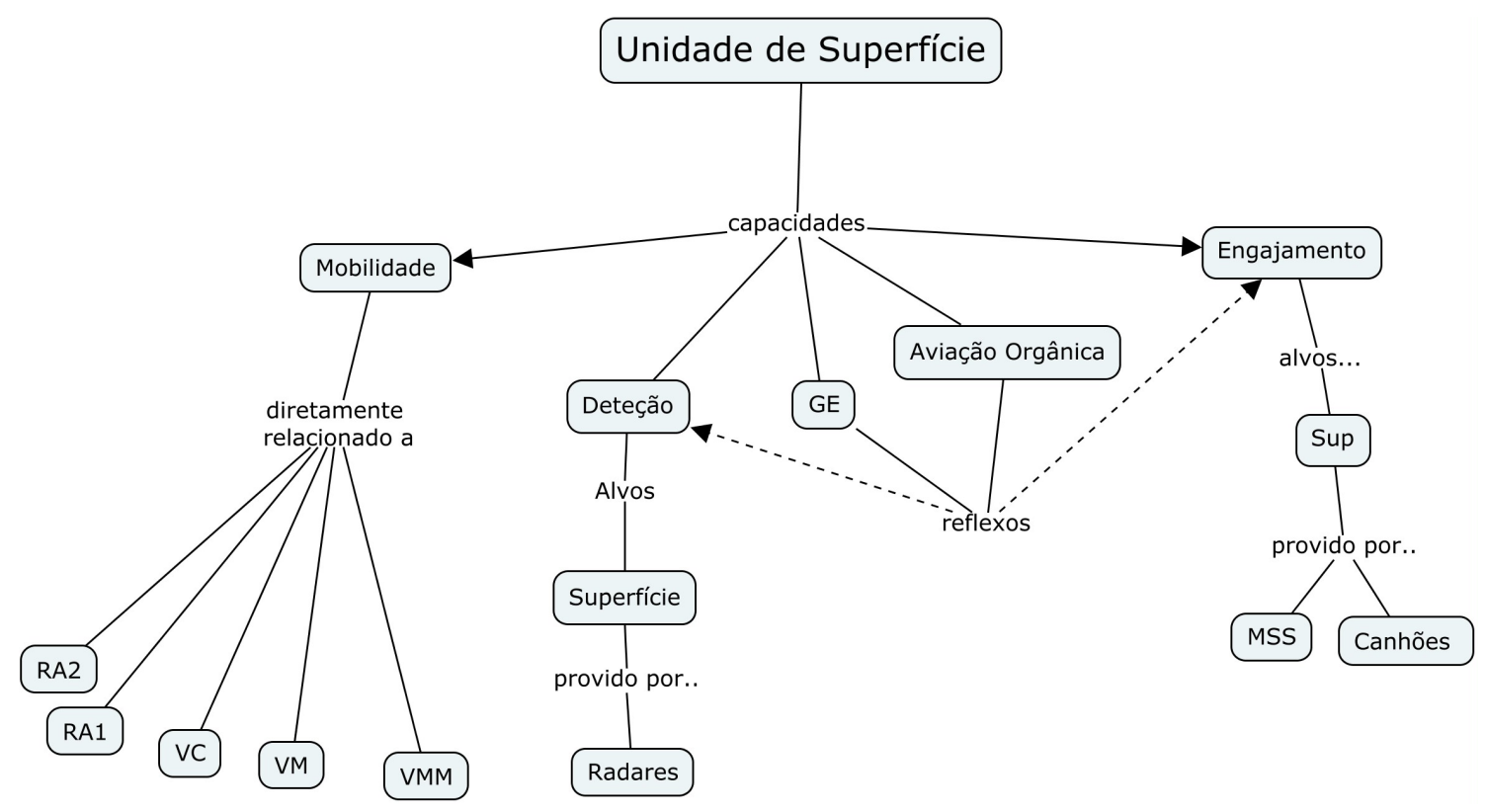

Figura 2: Estrutura conceitual dos critérios

sugerida ao decisor é de que os valores entre 0 e 1 representem condições de prontidão deteriorada do equipamento. Esta interpretação é coadunante com avaliação de condição de eficiência do equipamento, com conexão para a realidade do tomador de decisão.

É importante ressaltar que o objetivo almejado do trabalho é o de apresentar ao tomador de decisão uma função de preferência baseada nos critérios definidos, e não as problemáticas tradicionais de auxílio multicritério.

Portanto é importante que as alternativas sejam um grupo representativo das unidades a serem comparadas, mas que podem não apresentar a totalidade de unidades disponíveis. Os dados relativos às alternativas apresentadas são detalhados no apêndice.

\subsection{Avaliação Mobilidade}

Pela metodologia FITradeoff o primeiro passo é a ordenação pelo tomador de decisão dos critérios mais importantes. Para este problema a ordem estabelecida pelo tomador de decisão foi a seguinte: VM, VMM, RA2, VC e RA1.

Foram realizadas diversas interações, obtendo-se um esquema intermediário de limites de pesos apresentados na figura 3 .

A figura 3 estabelece os limites dos pesos para os critérios. Mais do que isto, possibilita ao tomador de decisão arbitrar entre os limites sendo escolhidos, sendo construída uma função de preferência coerente com as escolhas do decisor. Com base nos desempenhos das alternativas ainda candidatas, apresentada na figura 4. foi possível ao tomador de decisão efetuar a escolha pela alternativa 1 (em azul). 


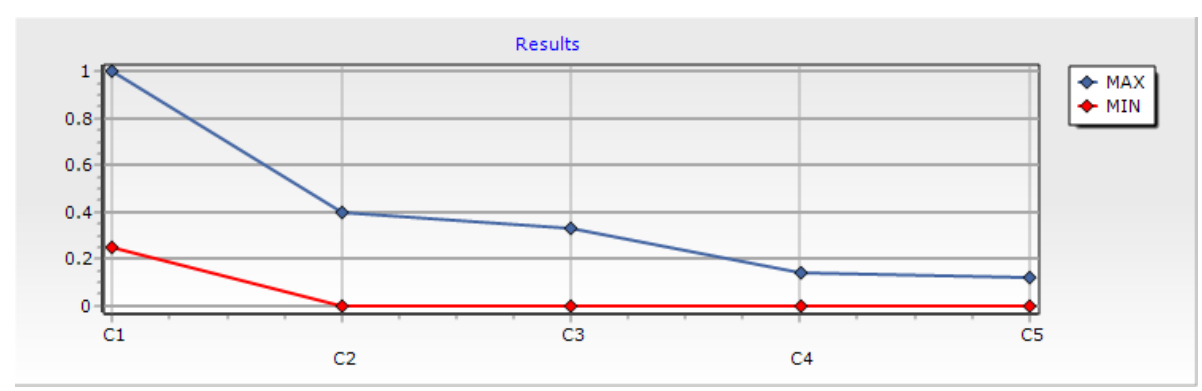

Figura 3: Limites de peso - eixo mobilidade

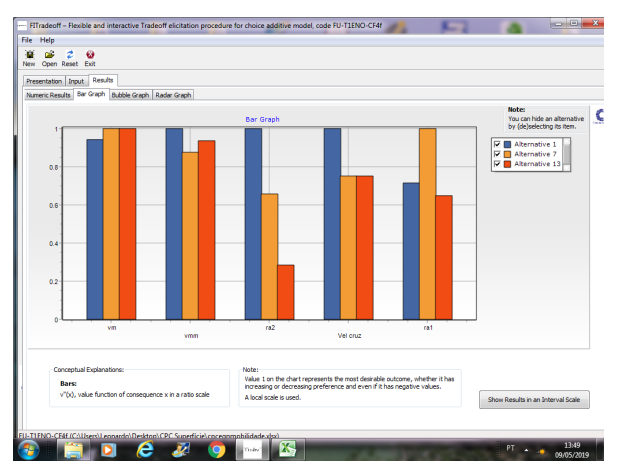

Figura 4: Limites de peso mobilidade

Escolhendo-se os valores das constantes de escala como os indicados pelo software como os mais favoráveis para esta alternativa, a saber: $k_{v m}=0,3752 ; k_{v m m}=$ 0,$3752 ; k_{r a 2}=0,1876 ; k_{v c}=0,0381 ;$ e $k_{r a 1}=0,0238$.

A função de preferência referente ao eixo mobilidade é determinada por:

$$
\begin{array}{r}
f(m o b)=0,3752 \frac{\left(V M-V M_{\min }\right)}{\left(V M_{\max }-V M_{\min }\right)}+0,3752 \frac{\left(V M M-V M M_{\min }\right)}{\left(V M M_{\max }-V M M_{\min }\right)}+ \\
0,1876 \frac{\left(R A 2-R A 2_{\min }\right)}{\left(R A 2_{\max }-R A 2_{\min }\right)}+0,0381 \frac{\left(R A 1-R A 1_{\min }\right)}{\left(R A 1_{\max }-R A 1_{\min }\right)}+ \\
0,0238 \frac{\left(V C-V C_{\min }\right)}{\left(V C_{\max }-V C_{\min }\right)}
\end{array}
$$

Note-se a importância da escolha de um conjunto de alternativas o qual represente toda a gama de valores, pois a normalização é condicionada entre os valores mínimos e máximos do desempenho das alternativas nos critérios. Também é importante que os valores da função de preferência mobilidade são parâmetros importantes para o tomador de decisão, quando forem realizadas a avaliação intereixos.

Sugere-se inclusive, que os valores das alternativas sejam utilizados como referência por ocasião das avaliações subsequentes.

\subsection{Avaliacão Detecão}


Para o eixo "deteção" foram considerados como critérios somente os equipamentos radar voltados para busca de superfície $(31,32,33,34,34 \mathrm{M}, 35,45 \mathrm{M}, 46 \mathrm{~A}$ e 46M). Suas características técnicas não serão apresentadas, mas durante a avaliação notou-se a importância do aspecto de alcance para escolha da preferência.

Foi utilizada a interpretação de que um intervalo contínuo seria admissível, considerando os valores intermediários como degradação do equipamento. A ordem de preferência dada foi: R35 R46A R34 R34M R46M R45M R33 R32 e R31.

Neste eixo a avaliação foi completada. A figura 5 apresenta os limites de constantes de escala encontrados

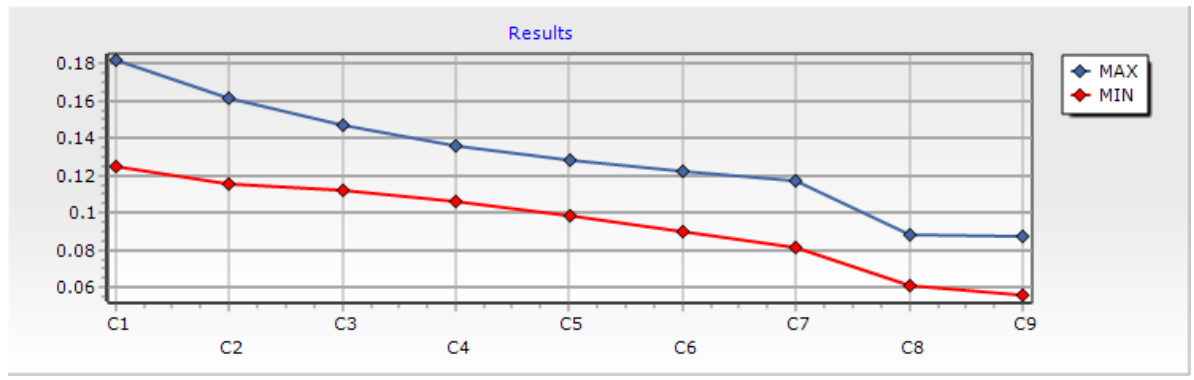

Figura 5: Limites de peso - eixo deteção

Foram arbitrados entre estes limites as seguintes constantes de escala, utilizando a normalização dos pontos médios entre os limites encontrados : $k_{R 35}=$ 0,$1444 ; k_{R 46 A}=0,1264 ; k_{R 34}=0,1264 ; k_{R 34 M}=0,1264 ; k_{R 46 M}=0,1258 ; k_{R 45 M}=$ 0,$11 ; k_{R 33}=0,963 ; k_{R 32}=0,0722 ; k_{R 31}=0,0722$

\subsection{Avaliação Engajamento}

Quanto ao eixo engajamento são considerados os canhões e os mísseis superfície superfície, levando-se em consideração suas respectivas dotações de bordo (quantidades). Em ambos os casos, o processo de escolha foi completado rapidamente, pois algumas alternativas possuem armamentos em quantidade e alcance significativamente superiores em relação às demais.

Desta forma muitos limites mínimos de constantes de escala igualam-se a zero, conforme pode ser observado nas figuras 6 e 7 .

De modo a possibilitar uma função de preferência com maior aderência ao propósito, foi utilizado um procedimento de escolha de constantes de escala intermediárias entre os limites, sendo estas normalizadas pela unidade, e posteriormente submetido a aprovação do tomador de decisão. Encontra-se em [7] utilização de procedimento similar.

Obtendo-se os seguintes resultados parciais:

- canhões: $k_{130 / 70}=0,4983 ; k_{115 / 45}=0,3185 ; k_{114 / 55}=0,07779 ; k_{100 / 55}=0,07195 ; e k_{127 / 54}=$ 0.0335 


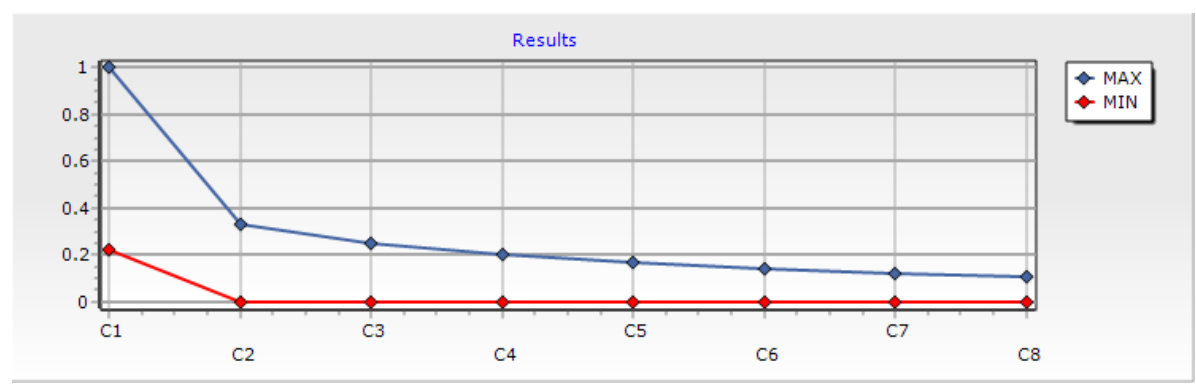

Figura 6: Limites de peso - critério misseis

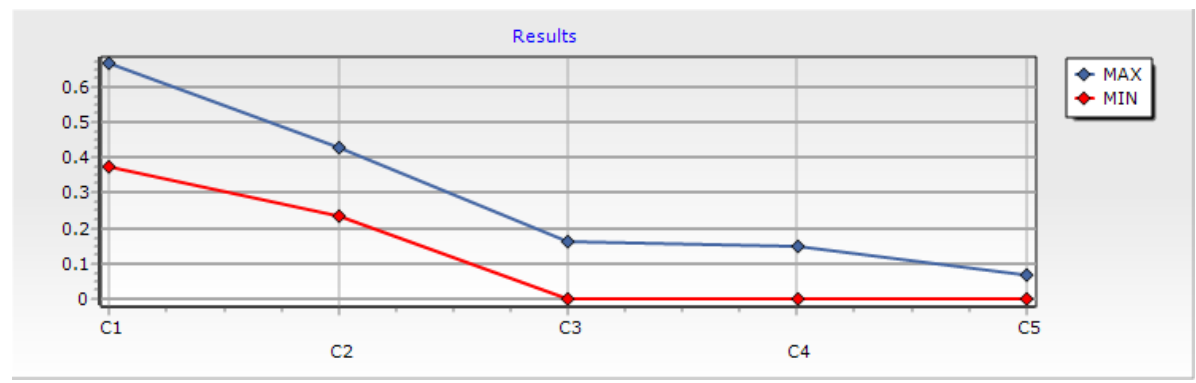

Figura 7: Limites de peso - critério canhões

- $\operatorname{misseis} \sup k_{M 2}=0,4717 ; k_{M 1}=0,1321 ; k M 3=0,1056 ; k_{M 4}=0,0755 ; k_{M 8}=$ 0,$06792 ; k_{M 7}=0,05660 ; k_{M 5}=0,04906 ; k_{M 6}=0,0415$

Para o exemplo utilizado, que tem como contexto uma fase inicial de confrontos, tendo como referência os preceitos das "Ações de Superfície", a comparação entre canhões e mísseis não é pertinente (a exceção em ações de defesa aeroespacial entre canhões e mísseis Ar-superfície). Destarte a capacidade de engajamento para este estudo de caso utiliza somente os valores da função de mísseis, podendo utilizar a elicitação de canhões para etapas futuras dos confrontos.

Posteriormente é realizado o procedimento de avaliação do eixo engajamento, a partir dos resultados parciais obtidos, apresentados na tabela 1 .

Destaca-se que a alternativa 9 obteve avaliação 0 no tocante a ambos critérios, face não possuir armamento voltado para o engajamento a unidades de superfície.

\subsection{Avaliação GE e Aeronave orgânica}

A avaliação do eixo aeronave orgânica, neste estudo de caso, avalia apenas a capacidade de operação de aeronaves embarcadas, dada pelo número de aeronaves orgânicas. Consiste, portanto, em apenas um critério, o qual consiste na avaliação do próprio eixo.

Quanto à capacidade de guerra eletrônica, note-se no apêndice que todas as alternativas possuem o mesmo equipamento o que impossibilita uma verificação direta pelo sistema do tradeoff, utilizando-se este critério. 


\begin{tabular}{lll}
\hline Alternativas & mísseis & canhões \\
\hline Alternativa 1 & 0,577358491 & 0,029749999 \\
Alternativa 2 & 0 & 0,077792226 \\
Alternativa 3 & 0,20754717 & 0,033468749 \\
Alternativa 4 & 0,124528302 & 0 \\
Alternativa 5 & 0,024528302 & 0,077792226 \\
Alternativa 6 & 0,041509434 & 0,035978905 \\
Alternativa 7 & 0,049056604 & 0 \\
Alternativa 8 & 0 & 0,498312479 \\
Alternativa 9 & 0 & 0 \\
Alternativa 10 & 0,024528302 & 0,077792226 \\
Alternativa 11 & 0,041509434 & 0 \\
Alternativa 12 & 0,024528302 & 0,318468737 \\
Alternativa 13 & 0,016509434 & 0,033468749 \\
Alternativa 14 & 0,049056604 & 0,033468749 \\
Alternativa 15 & 0 & 0,071957809 \\
\hline
\end{tabular}

Tabela 1: Engajamento

Dois caminhos podem ser encarados, o primeiro excluir este equipamento como um critério, o que permitiria a construção de uma função parcial incompleta. E o segundo, a criação de uma alternativa adicional, representada como alternativa "dummy", sem o equipamento em questão, apenas para consecução da avaliação.

Futuros trabalhos podem analisar mais detalhadamente estes eixos, estabelecendo critérios específicos relacionados à colaboração ao engajamento e deteção.

\subsection{Avaliação Intercritérios}

Com base nas avaliações obtidas de cada eixo, é possível a comparação entre os eixos obtendo uma função de funcões de preferência. A tabela 2 apresenta os resultados das alternativas obtidos em cada eixo.

Seguindo o método, a ordenação dos eixos estabecida foi a seguinte: ANV; deteção; CAPGE, Engajamento e Mobilidade.

Após a elicitação flexível, foi determinado o conjunto de restrições às constantes de escala, conforme apresentado na figura 8.

Obtendo-se o seguinte conjunto de constantes de escala, tidas como ótimas para a alternativa 3, que obteve o melhor desempenho global segundo as avaliações do decisor.

0

$$
k_{\text {anv }}=0,9677 ; k_{\text {det }}=0,0302 ; \text { kcapge }=0,0019 ; k_{\text {engajamento }}=0,0001 ; k_{\text {mobilidade }}=
$$

Estes pesos refletem os conceitos das ações de superfície, dentro do contexto de um confronto inicial, dentro da avaliação do tomador de decisão. Destaca-se que, 


\begin{tabular}{llllll}
\hline Alternativas & mobilidade & deteção & engajamento & GE & ANV \\
\hline Alternativa 1 & 0,921612587 & 0,0722 & 0,577358491 & 1 & 2 \\
Alternativa 2 & 0,494638192 & 0,3966 & 0 & 1 & 1 \\
Alternativa 3 & 0,660861349 & 0,3327 & 0,20754717 & 1 & 2 \\
Alternativa 4 & 0,640492929 & 0,2364 & 0,124528302 & 1 & 0 \\
Alternativa 5 & 0,455276569 & 0,2364 & 0,024528302 & 1 & 1 \\
Alternativa 6 & 0,093207635 & 0,2364 & 0,041509434 & 1 & 0 \\
Alternativa 7 & 0,73241841 & 0,1444 & 0,049056604 & 1 & 1 \\
Alternativa 8 & 0,642542492 & 0,1264 & 0 & 1 & 1 \\
Alternativa 9 & 0,546171003 & 0,1685 & 0 & 1 & 2 \\
Alternativa 10 & 0,48432855 & 0,2522 & 0,024528302 & 1 & 1 \\
Alternativa 11 & 0,47769827 & 0,2943 & 0,041509434 & 1 & 2 \\
Alternativa 12 & 0,267115985 & 0,2522 & 0,024528302 & 1 & 1 \\
Alternativa 13 & 0,719622464 & 0,3327 & 0,016509434 & 1 & 1 \\
Alternativa 14 & 0,584831393 & 0,3244 & 0,049056604 & 1 & 0 \\
Alternativa 15 & 0,29021635 & 0,2522 & 0 & 1 & 0 \\
Dummy & 0,29021635 & 0,198 & 0 & 0 & 0 \\
\hline
\end{tabular}

Tabela 2: Dados avaliação inter-eixos

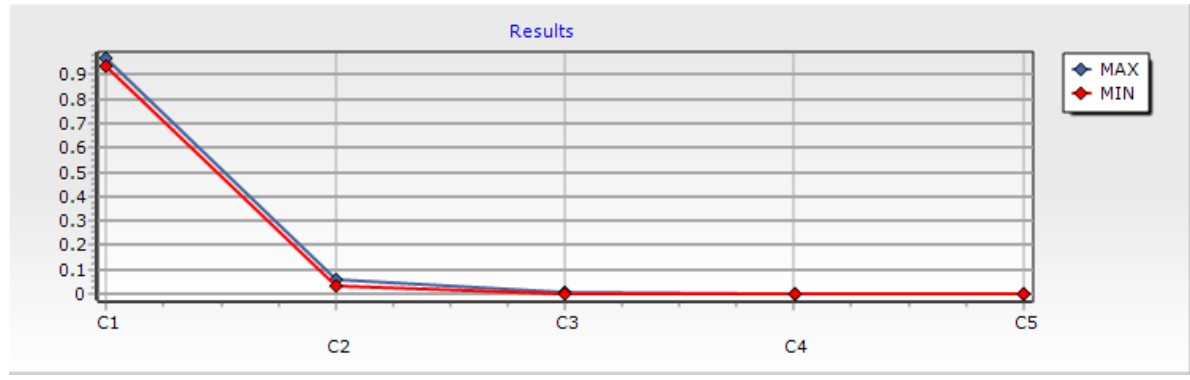

Figura 8: Limites de peso - intereixos

neste momento, para o tomador de decisão o eixo mobilidade torna-se irrelevante frente aos outros eixos.

Torna-se possível estabelecer a função de preferências referentes às capacidades, utilizando-se as constantes de escala elicitadas para a comparação da hierarquia intereixos neste contexto:

$$
\begin{aligned}
& f(\text { intereixos })=0,9677 \frac{\left(A N V-A N V_{\min }\right)}{\left(A N V_{\max }-A N V_{\min }\right)}+0,0302 \frac{\left(\operatorname{det}-\operatorname{det}_{\min }\right)}{\left(\operatorname{det}_{\max }-\operatorname{det}_{\min }\right)}+
\end{aligned}
$$

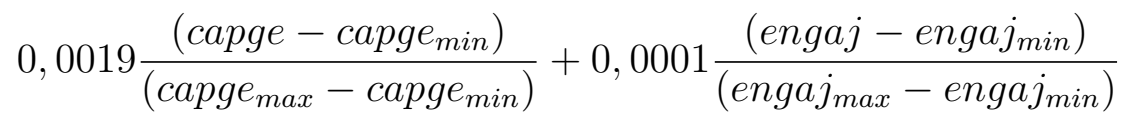

Todavia, diferentes momentos do confronto podem demandar novos julgamentos, podendo alterar significativamente o conjunto de constantes de escala, em consonância com o caráter cíclico do planejamento militar. 


\section{CONCLUSÃO}

O presente trabalho apresenta uma prova de conceito da utilização de metologia FITradeoff para realização de CPC de unidades de superfície, especificamente escoltas em emprego de superfície. Esta proposta é inovadora na Marinha do Brasil, com aplicações que auxiliam o processo decisório militar.

Como contribuição metodológica, além de estabelecer uma proposta viável para a realização da CPC de unidades de superfíce, segundo a situação proposta, estende o uso da metodologia FITradeoff para um uso diverso das problemáticas tradicionais multicritério, onde o foco está na obtenção da função de preferência em vez de seleção, classificação ou ordenação das alternativas.

Adicionalmente o trabalho apresenta uma estrutura hierárquica de critérios para execução da CPC por unidades de superfície, comprovando a viabilidade da utilização hierárquica do FITradeoff.

Futuros trabalhos podem contribuir para a extensão do modelo, realizando a análise dos critérios componentes dos eixos GE, deteção e engajamento, com base em desempenho. O ideal seria que a elicitação utilizasse o modelo de ordenação, recentemente proposto por [8], o qual também pode ser explorado em outra oportunidade.

\section{AGRADECIMENTOS}

Os autores agradecem a valiosa contribuição dos Capitães de Fragata EWERTON RODRIGUES CALFA, LEONARDO BARBOSA SOUZA e ALEXANDER THOMAZ ARRUDA, da Escola de Guerra Naval, os quais representaram, com sua experiência, os papéis do tomador de decisão 


\section{REFERÊNCIAS BIBLIOGRÁFICAS}

[1] COSTA, H. G.; ROBOREDO, M. G.; PESSôA, L. A. M. Metodologias Multicritério no campo militar: um panorama do domínio tático/operacional. 2019. 2

[2] De Almeida, A. T. et al. A new method for elicitation of criteria weights in additive models: Flexible and interactive tradeoff. European Journal of Operational Research, Elsevier Ltd., v. 250, n. 1, p. 179-191, 2016. ISSN 03772217. Disponível em: <http://dx.doi.org/10.1016/j.ejor.2015.08.058>. 2

[3] BOTELHO, T. A. T. et al. Aplicação do método multicritério FITradeoff para escolha de obuseiro para batalhão de artilharia de Fuzileiros Navais. In: XLIX Simpósio Brasileiro de Pesquisa Operacional. [S.l.: s.n.], 2017. 2

[4] PESSÔA, L. A. M.; FERREIRA, R. J. P.; ALMEIDA, A. T. de. Análise de escolha de armamento naval baseado no método multicritério FITradeoff. In: XLVIII SBPO Simpósio Brasileiro de Pesquisa Operacional. [S.l.: s.n.], 2016. p. 40534061. 2

[5] BRASIL. Manual de Planejamento Operativo da Marinha. Processo de Planejamento Militar, vol I. Brasília: Estado Maior da Armada, 2006. 3

[6] BRASIL. Doutrina de Operações Conjuntas, II volume. Brasília: Ministério da Defesa, 2011. 3

[7] PESSÔA, L. A. M. et al. Avaliação de impacto de política de decisão: uma proposta utilizando o Fitradeoff. In: . Maceió: ABEPRO, 2018. Disponível em: $<$ http:/ $/$ www.abepro.org.br/publicacoes/artigo.asp? $=$ enegep\&a $=2018 \& c=36580>$. 5., 8

[8] FREJ, E. A.; ALmeidA, A. T. de; COSTA, A. P. C. S. Using data visualization for ranking alternatives with partial information and interactive tradeoff elicitation. Operational Research, jan. 2019. ISSN 1866-1505. Disponível em: <https://doi.org/10.1007/s12351-018-00444-2>. 12

\section{A. DADOS DAS ALTERNATIVAS}




\begin{tabular}{llllll}
\hline Alternativas & VC & VMM & VM & RA1 & RA2 \\
\hline Alternativa 1 & 20 & 32 & 33 & 5500 & 4560 \\
Alternativa 2 & 18 & 28 & 30 & 4000 & 1690 \\
Alternativa 3 & 18 & 30 & 31 & 7000 & 2400 \\
Alternativa 4 & 12 & 31 & 33 & 2500 & 755 \\
Alternativa 5 & 15 & 28 & 30 & 3800 & 1200 \\
Alternativa 6 & 15 & 23 & 24 & 3100 & 2300 \\
Alternativa 7 & 15 & 28 & 35 & 7700 & 3000 \\
Alternativa 8 & 15 & 30 & 32 & 7500 & 1580 \\
Alternativa 9 & 20 & 28 & 29 & 4580 & 3180 \\
Alternativa 10 & 15 & 28 & 30 & 5300 & 1650 \\
Alternativa 11 & 18 & 28 & 30 & 4500 & 1300 \\
Alternativa 12 & 12 & 26 & 27 & 4500 & 1375 \\
Alternativa 13 & 15 & 30 & 35 & 5000 & 1300 \\
Alternativa 14 & 18 & 30 & 31 & 4500 & 1090 \\
Alternativa 15 & 15 & 24 & 25 & 7500 & 4350 \\
\hline
\end{tabular}

Tabela 3: Dados eixo mobilidade

\begin{tabular}{lllllllll}
\hline Alternativas & M1 & M2 & M3 & M4 & M5 & M6 & M7 & M8 \\
\hline Alternativa 1 & 0 & 45 & 8 & 0 & 0 & 0 & 0 & 0 \\
Alternativa 2 & 0 & 0 & 0 & 0 & 0 & 0 & 0 & 0 \\
Alternativa 3 & 8 & 0 & 0 & 40 & 0 & 0 & 0 & 0 \\
Alternativa 4 & 0 & 0 & 0 & 0 & 0 & 0 & 4 & 6 \\
Alternativa 5 & 0 & 0 & 0 & 0 & 4 & 0 & 0 & 0 \\
Alternativa 6 & 0 & 0 & 0 & 0 & 0 & 4 & 0 & 0 \\
Alternativa 7 & 0 & 0 & 0 & 0 & 8 & 0 & 0 & 0 \\
Alternativa 8 & 0 & 0 & 0 & 0 & 0 & 0 & 0 & 0 \\
Alternativa 9 & 0 & 0 & 0 & 0 & 0 & 0 & 0 & 0 \\
Alternativa 10 & 0 & 0 & 0 & 0 & 4 & 0 & 0 & 0 \\
Alternativa 11 & 0 & 0 & 0 & 0 & 0 & 4 & 0 & 0 \\
Alternativa 12 & 0 & 0 & 0 & 0 & 4 & 0 & 0 & 0 \\
Alternativa 13 & 1 & 0 & 0 & 0 & 0 & 0 & 0 & 0 \\
Alternativa 14 & 0 & 0 & 0 & 0 & 8 & 0 & 0 & 0 \\
Alternativa 15 & 0 & 0 & 0 & 0 & 0 & 0 & 0 & 0 \\
\hline
\end{tabular}

Tabela 4: Dados eixo engajamento - superfície-mísseis 


\begin{tabular}{llllll}
\hline Alternativas & $\mathbf{1 1 4 / 5 5}$ & $\mathbf{1 1 5 / 4 5}$ & $\mathbf{1 2 7 / 5 4}$ & $\mathbf{1 3 0 / 7 0}$ & $\mathbf{1 0 0 / 5 5}$ \\
\hline Alternativa 1 & 0 & 0 & 400 & 0 & 0 \\
Alternativa 2 & 250 & 0 & 0 & 0 & 0 \\
Alternativa 3 & 0 & 0 & 450 & 0 & 0 \\
Alternativa 4 & 0 & 0 & 0 & 0 & 0 \\
Alternativa 5 & 250 & 0 & 0 & 0 & 0 \\
Alternativa 6 & 0 & 0 & 0 & 0 & 600 \\
Alternativa 7 & 0 & 0 & 0 & 0 & 0 \\
Alternativa 8 & 0 & 0 & 0 & 900 & 0 \\
Alternativa 9 & 0 & 0 & 0 & 0 & 0 \\
Alternativa 10 & 250 & 0 & 0 & 0 & 0 \\
Alternativa 11 & 0 & 0 & 0 & 0 & 0 \\
Alternativa 12 & 0 & 800 & 0 & 0 & 0 \\
Alternativa 13 & 0 & 0 & 450 & 0 & 0 \\
Alternativa 14 & 0 & 0 & 450 & 0 & 0 \\
Alternativa 15 & 0 & 0 & 0 & 0 & 1200 \\
\hline
\end{tabular}

Tabela 5: Dados eixo engajamento - canhões

\begin{tabular}{|c|c|c|c|c|c|c|c|c|c|c|c|c|c|c|c|}
\hline Alternativas & 32 & 31 & 33 & 34 & $34 \mathrm{M}$ & 35 & $37 \mathrm{~A}$ & 37B & $38 \mathrm{M}$ & 39 & $41 \mathrm{~A}$ & 43 & $45 \mathrm{M}$ & $46 \mathrm{M}$ & $46 \mathrm{~A}$ \\
\hline Alternativa 1 & 1 & 0 & 0 & 0 & 0 & 0 & 0 & 0 & 1 & 0 & 0 & 0 & 0 & 0 & 0 \\
\hline Alternativa 2 & 0 & 0 & 0 & 0 & 1 & 1 & 0 & 0 & 1 & 0 & 0 & 0 & 0 & 1 & 0 \\
\hline Alternativa 3 & 0 & 0 & 1 & 0 & 1 & 0 & 0 & 1 & 0 & 0 & 0 & 1 & 1 & 0 & 0 \\
\hline Alternativa 4 & 0 & 0 & 0 & 0 & 1 & 0 & 0 & 0 & 0 & 0 & 0 & 0 & 1 & 0 & 0 \\
\hline Alternativa 5 & 0 & 0 & 0 & 1 & 0 & 0 & 0 & 0 & 0 & 0 & 0 & 0 & 1 & 0 & 0 \\
\hline Alternativa 6 & 0 & 0 & 0 & 0 & 1 & 0 & 0 & 0 & 0 & 0 & 0 & 0 & 1 & 0 & 0 \\
\hline Alternativa 7 & 0 & 0 & 0 & 0 & 0 & 1 & 0 & 0 & 0 & 0 & 1 & 0 & 0 & 0 & 0 \\
\hline Alternativa 8 & 0 & 0 & 0 & 0 & 0 & 0 & 0 & 0 & 0 & 0 & 1 & 1 & 0 & 0 & 1 \\
\hline Alternativa 9 & 1 & 0 & 1 & 0 & 0 & 0 & 0 & 0 & 0 & 1 & 0 & 0 & 0 & 0 & 0 \\
\hline Alternativa 10 & 0 & 0 & 0 & 1 & 0 & 0 & 0 & 0 & 0 & 0 & 0 & 0 & 0 & 1 & 0 \\
\hline Alternativa 11 & 0 & 1 & 1 & 0 & 0 & 0 & 0 & 0 & 0 & 0 & 0 & 0 & 0 & 1 & 0 \\
\hline Alternativa 12 & 0 & 0 & 0 & 0 & 1 & 0 & 1 & 0 & 0 & 0 & 0 & 0 & 0 & 1 & 0 \\
\hline Alternativa 13 & 0 & 0 & 1 & 0 & 1 & 0 & 0 & 0 & 0 & 0 & 0 & 0 & 1 & 0 & 0 \\
\hline Alternativa 14 & 0 & 1 & 0 & 1 & 0 & 0 & 0 & 0 & 0 & 0 & 0 & 0 & 0 & 1 & 0 \\
\hline Alternativa 15 & 0 & 0 & 0 & 0 & 1 & 0 & 0 & 0 & 0 & 0 & 0 & 0 & 0 & 1 & 0 \\
\hline
\end{tabular}

Tabela 6: Dados eixo deteção - radares 